\title{
Present situation of antimicrobial resistance in Korea
}

\begin{abstract}
Resistance of bacteria to antimicrobial agents is a worldwide concern. In Korea, resistant bacteria are more prevalent than in other industrialized countries. Methicillin-resistant Staphylococcus aureus, erythromycinresistant Streptococcus pyogenes, penicillin non-susceptible pneumococci, $\beta$-lactamase-producing gonococci, extendedspectrum $\beta$-lactamase (ESBL)-producing Escherichia coli and Klebsiella pneumoniae, class $\mathrm{C} \beta$-lactamase-producing E. coli, fluoroquinolone-resistant E. coli, and aminoglycoside-resistant Acinetobacter baumannii and Pseudomonas aeruginosa are examples of resistant bacteria prevalent in Korea, and their presence suggests a high level of antimicrobial selective pressure and the nosocomial spread of resistant bacteria. Recently observed rapid increases in the incidence of vancomycin-resistant Enterococcus faecium and carbapenem-resistant $P$. aeruginosa present new threats in Korea.
\end{abstract}

Key words Antimicrobial resistance in Korea $\cdot$ Resistance survey $\cdot$ Nosocomial pathogen

\section{Introduction}

It was in the early 1990s that prominent scientists viewed resistance to antimicrobial agents as a crisis and calamity. ${ }^{1,2}$ They insisted that the extensive use of antibiotics in the community and hospitals fueled the crisis in antibiotic resistance, which resulted in virtually all pathogenic bacteria

Y. Chong $(\bowtie) \cdot$ K. Lee

Department of Clinical Pathology, Research Institute of Bacterial Resistance, Yonsei University College of Medicine, C.P.O. Box 8044 , Seoul, Korea

Tel. +82-2-361-5866; Fax +82-2-313-0908

e-mail: whonetkor@yumc.yonsei.ac.kr becoming resistant to older antibiotics. During the past several years, the antibiotic resistance problem has worsened noticeably in Korea. The increased prevalence of known resistant organisms and the emergence of newly resistant, organisms such as vancomycin-resistant enterococci (VRE), extended-spectrum $\beta$-lactamase (ESBL)-producing Escherichia coli and Klebsiella pneumoniae, imipenemresistant Pseudomonas aeruginosa, and fluoroquinoloneresistant gram-negative bacilli, have become of real concern.

The methods used to test antimicrobial susceptibility used may affect the result. In Korea, the majority of laboratories use the disk diffusion method, although some large hospitals use the commercial broth microdilution test. The disk diffusion method used is the only National Committee for Clinical Laboratory Standards (NCCLS) approved method, except for some antimicrobial agents for which NCCLS breakpoints are not available. ${ }^{3}$ Most reports on antimicrobial resistance in Korea were based on isolates in large tertiary care hospitals. Therefore, the resistance rates were influenced by the prevalence of nosocomial infections. The analysis of resistance of isolates from outpatients may also fail to show true resistance rates of community-acquired pathogens, as some of the outpatients may have acquired the organisms in hospitals.

Before presenting the current resistance status of some of the more important pathogens, we briefly present the trend of resistance in the recent past in a tertiary care hospital, together with the resistance pattern of Neisseria gonorrhoeae from other studies, to show the general picture of resistance in Korea (Figs. 1, 2). Trends show that methicillin-resistant Staphylococcus aureus (MRSA), penicillin-resistant Streptococcus pneumoniae, ampicillinresistant Haemophilus influenzae, and piperacillin-resistant Pseudomonas aeruginosa were already prevalent in the early 1990s, and all of their resistance rates were over $50 \%$ in 1999. During this period, VRE, ESBL-producing $K$. pneumoniae, cefoxitin-resistant $E$. coli, imipenem-resistant $P$. aeruginosa, and fluoroquinolone-resistant $E$. coli have emerged and their resistance rates have increased significantly. 


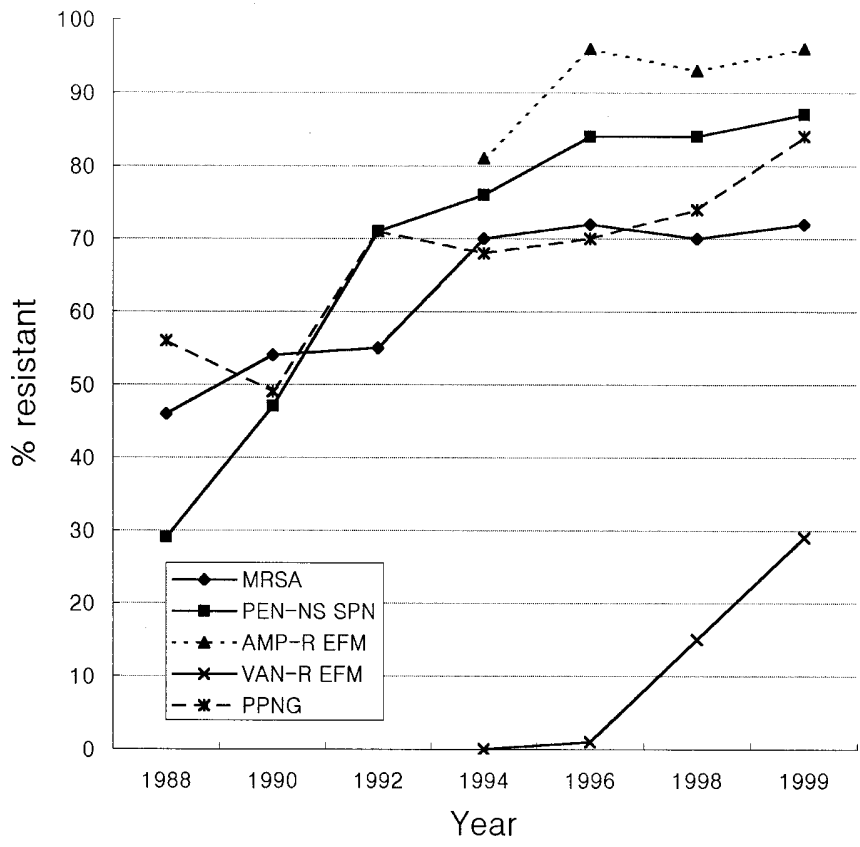

Fig. 1. Trends in antimicrobial resistance of gram-positive and gramnegative cocci between 1988 and 1999 in a tertiary care hospital in Korea. The resistance rates of Neisseria gonorrhoeae are cited from other studies. MRSA, Methicillin-resistant Staphylococcus aureus; PEN-NSSPN, penicillin-nonsusceptible Streptococcus pneumoniae AMP-R EFM, ampicillin-resistant Enterooccus faecium; VAN-R EFM, vancomycin-resistant $E$. faecium; PPNG, penicillinase-producing Neisseria gonorrhoeae

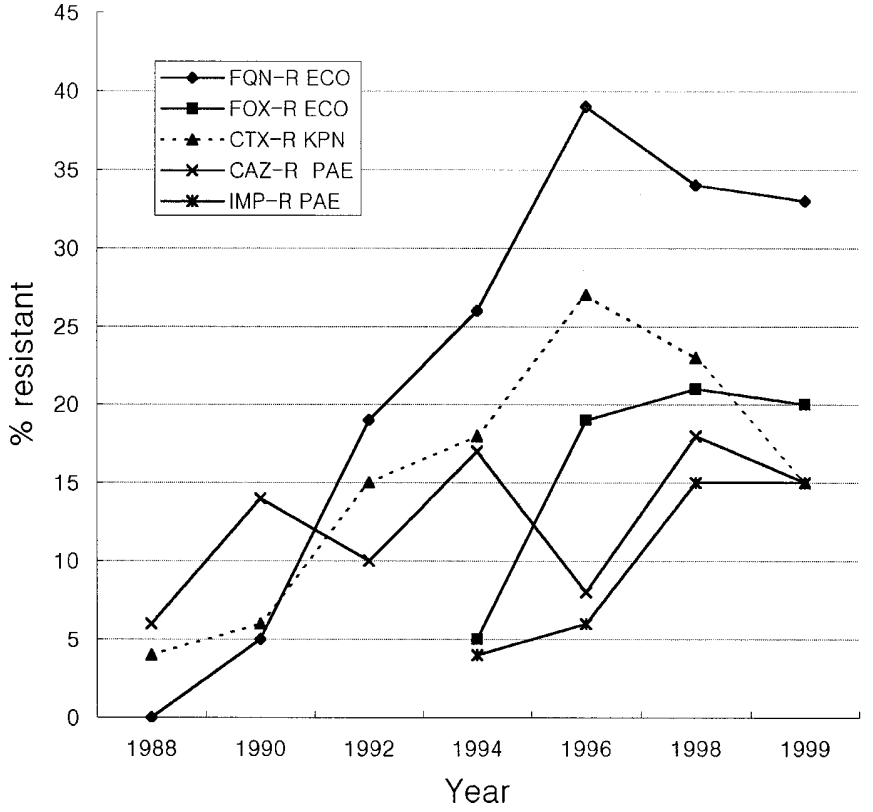

Fig. 2. Trends in antimicrobial resistance of gram-negative bacilli between 1988 and 1999 in a tertiary care hospital in Korea. FON-R ECO, Fluoroquinolone-resistant Escherichia coli; FOX-R ECO, cefoxitinresistant E. coli; $C T X-R \quad K P N$, cefotaxime-resistant Klebsiella pneumoniae; CAZ-R PAE, ceftazidime-resistant Pseudomonas aeruginosa; IMP-R PAE, imipenem-resistant $P$. aeruginosa

Table 1. Antimicrobial resistance rates of important aerobic gram-positive cocci isolated in 1998 in Korea

\begin{tabular}{lll}
\hline Species (no. tested) & Antimicrobial agents & Resistance rate (\%) \\
\hline Staphylococcus aureus (26042) & Oxacillin & 72 \\
& Clindamycin & 63 \\
& Fluoroquinolone & 66 \\
Streptococcus pyogenes (41) & Cotrimoxazole & 3 \\
S. agalactiae (40) & Erythromycin & 27 \\
S. pneumoniae (2187) & Erythromycin & 26 \\
Enterococcus faecium (2968) & Penicillin non-susceptible & 78 \\
E. faecalis $(7075)$ & Ampicillin & 80 \\
& Vancomycin & 11 \\
C.incomycin & Vancomy
\end{tabular}

${ }^{a}$ Cited from references 5 and $9 ; S$. pyogenes and $S$. agalactiae were isolated in the period 1993-1996

\section{Resistance of staphylococci}

S. aureus is an important pathogen, frequently isolated from both outpatients and inpatients with various infections. The increased prevalence of MRSA, a typical nosocomial pathogen, is a serious problem, because it is resistant to all existing $\beta$-lactam antibiotics. In Korea, MRSA was not detected in the 1960s. During the period 1971-1979, 3\% to $9 \%$ of $S$. aureus isolates from blood were methicillinresistant, but in 1980 the rate was $25 \% .{ }^{4}$ In the same hospital, the methicillin-resistance rates of all $S$. aureus rose sharply, to $44 \%$, in 1988 and gradually rose again to reach around $70 \%$ in 1995 . In a 1998 Korean survey, the methicillin resis- tance rate was found to be $72 \%$ (Table 1$).^{5}$ In a study of strains from wounds, those isolated in the 1990s were found to be highly resistant, with a methicillin minimum inhibitory concentration for $50 \%$ of isolates $\left(\mathrm{MIC}_{50}\right)$ of $512 \mu \mathrm{g} / \mathrm{ml}$. It was also noted that the prevalence of coagulase type II increased to $30 \%$ and that most were expression class 3 or 4 . $^{6}$ In Korea, arbekacin is not used, and a 1996 study showed that all of the isolates tested were susceptible to this drug. ${ }^{7}$

The emergence of a vancomycin-resistant (intermediate) S. aureus (VISA) in 1996 in Japan, caused new concerns. Studies were undertaken to search for VISA in several Korean hospitals. Kim et al. ${ }^{8}$ identified a VISA isolate among MRSAs isolated from a pelvic abscess specimen of a 45-year-old man. However, other workers were not able to 
detect any VISA isolates. We screened for the presence of Mu3 type strains, using Mu3 agar, and found that $15 \%$ of the isolates showed satellitism around the aztreonam disk. Population analysis demonstrated that they were not VISA strains, but contained subpopulations which were resistant to vancomycin, indicating their potential to become VISA.

\section{Resistance of streptococci and enterococci}

In Korea, Streptococcus pyogenes remained very susceptible to $\beta$-lactam antibiotics, i.e., the MIC of penicillin G was $\leq 0.015 \mu \mathrm{g} / \mathrm{ml}$, while the resistance rate to erythromycin was $27 \%$ (Table 1 ) and to that tetracycline was $56 \%{ }^{9}$ The resistance rates to these drugs in Korea were much higher than the $2.7 \%$ and $16.5 \%$ found in Japan during the early 1990s. ${ }^{10}$

S. agalactiae neonatal infection is much less frequent in Korea than in the United States, probably because of the lower genital carriage rate, $5.9 \%$, in Korean pregnant women. ${ }^{11}$ The prevalent serotypes remain Ia, Ib, and III, while new serotypes, VI and VIII, have been detected recently. ${ }^{9}$ S. agalactiae is between five-fold and eight-fold less susceptible to penicillin than S. pyogenes. In our study, the MIC range of penicillin G for S. agalactiae was 0.03 $0.06 \mu \mathrm{g} / \mathrm{ml} .^{9}$ The resistance rate to erythromycin was $26 \%$ (Table 1) and that to tetracycline was $100 \%$, rates which were much higher than those in Japan in 1986-1992 (i.e., $1.2 \%$ and $42 \%$, respectively). ${ }^{10}$

S. pneumoniae is an important pathogen that causes a variety of infections, some of which are serious. The increase in penicillin G-non-susceptible pneumococci has become a serious problem in many parts of the world. ${ }^{12}$ The penicillin non-susceptible rate in a Korean tertiary care hospital rose from $3 \%$ in 1986 to $29 \%$ in 1988 , and then increased to $77 \%$ in $1993 .{ }^{13}$ Similar results have been reported in other hospitals. ${ }^{14,15}$ Capsular types $19 \mathrm{~F}$ and $23 \mathrm{~F}$ were frequently isolated, and these types of isolates were often non-susceptible to penicillin G. A Korean nationwide survey in 1998 showed the mean penicillin non-susceptible rate to be $78 \%$ (Table 1), which was much higher than the $52 \%$ reported in Japan during 1994-1995. ${ }^{16}$

Enterococci became more frequently isolated from inpatients, and the proportion of Enterococcus faecium increased to $38 \%$ of all enterococci in Korean tertiary hospitals. The ampicillin-resistance rate of $E$. faecalis is very low, but the ampicillin-resistance rate of E. faecium was $80 \%$ in 1998 (Table 1), and $96 \%$ in 1999 in a tertiary care hospital (Fig. 1). Although most enterococci were isolated from urine specimens, some were isolated from blood specimens. As enterococci are inherently less susceptible to $\beta$-lactam antibiotics, enterococcal endocarditis is treated with aminoglycoside combinations. However, a gradual increase in high-level aminoglycoside-resistant isolates has been noted. In a Korean tertiary care hospital in the period 1991-1992, high-level resistance rates to gentamicin were $20 \%$ in E. faecalis and $59 \%$ in E. faecium. ${ }^{17}$ In another study, the resistance rates to gentamicin and streptomycin were: E. faecalis, $61 \%$ and $39 \%$, respectively; and $E$. faecium, $45 \%$ and $55 \%$, respectively. ${ }^{18}$

The first isolation of VRE in Korea was reported in $1992,{ }^{19}$ but it was rare. In a tertiary care hospital, among the 31 E. faecalis and 11 E. faecium strains isolated from blood during the period 1996-1998, only one isolate of E. faecium was resistant to vancomycin. ${ }^{18}$ However, in another tertiary care hospital, the proportion of vancomycin-resistant $E$. faecium started to rise sharply in 1998 (Fig. 1) and reached $29 \%$ in 1999 . Most of the VRE were E. faecium of type VanA. Pulsed-field gel electrophoresis (PFGE) patterns suggested that both the clonal and the horizontal spread of resistance were the cause of the sharp increase. Donskey et $\mathrm{al}^{20}{ }^{20}$ noted that it was difficult to control the spread of VRE by infection control measures alone.

\section{Resistance of Neisseria and Moraxella}

Low-level penicillin G-resistant isolates of $N$. gonorrhoeae were already prevalent in Korea in the 1960s, but a penicillinase-producing strain (PPNG) was first reported in 1979. In the 1990s, almost all isolates were resistant to penicillin $G$, either by chromosomal resistance or by $\beta$ lactamase production (Table 2). Since the early 1990s, the proportion of PPNG has been to over $70 \%$ (Fig. 1). ${ }^{21,22}$ However, the isolates remained highly susceptible to ceftriaxone, i.e., the MIC range was $\leq 0.008-0.06 \mu \mathrm{g} / \mathrm{ml}$. A recent decrease in PPNG in other countries is probably related to the increase in quinolone-resistant strains, as reported in Hong Kong. ${ }^{23}$ In Japan, the ciprofloxacin resistance rate of gonococci isolated in the period 1997-1998 was

Table 2. Antimicrobial susceptibility of gonococci isolated in 1997-1999 in Korea

\begin{tabular}{lllllr}
\hline $\begin{array}{l}\text { Antimicrobial } \\
\text { agents }\end{array}$ & $\begin{array}{l}\text { Strains } \\
(\text { no. tested })\end{array}$ & $\begin{array}{l}\text { MIC range } \\
(\mu \mathrm{g} / \mathrm{ml})\end{array}$ & \multicolumn{2}{l}{ Percentage of isolates } \\
\cline { 5 - 6 } & & & Susceptible & Intermediate & Resistant \\
\hline Penicillin G & Non-PPNG (29) & $0.12-1$ & 0 & 100 & 0 \\
& PPNG (144) & $2->128$ & 0 & 0 & 100 \\
Ciprofloxacin & Non-PPNG (29) & $\leq 0.008-0.5$ & 59 & 41 & 0 \\
& PPNG (144) & $\leq 0.008-1$ & 35 & 64 & 1 \\
Tetracycline & All (173) & $1-8$ & 0 & 13 & 87 \\
\hline
\end{tabular}


$23.6 \%{ }^{24}$ while the ciprofloxacin resistance rate of PPNG was only $4 \%$ in $1994 .{ }^{25}$ In Korea, isolates of gonococci often showed intermediate resistance to ciprofloxacin (i.e., MICs were $\leq 1 \mu \mathrm{g} / \mathrm{ml}$ ) until 1999, but high-level resistant isolates started to be isolated in 2000. Spectinomycin-resistant gonococci remained rare, as was reported in other Asian countries. ${ }^{25}$ Moraxella (Branhamella) catarrhalis is not frequently isolated from clinical specimens in Korea. Studies showed that approximately $95 \%$ of recent isolates were producing $\beta$-lactamase.

\section{Resistance of gram-negative bacilli to $\beta$-lactams}

Enterobacteriaceae and glucose-nonfermenting gramnegative bacilli are frequently isolated from various community-acquired and nosocomial infections. Some of the current resistance problems with Enterobacteriaceae are an increase in the numbers of ESBL- or AmpC $\beta$ lactamase-producing strains of E. coli and $K$. pneumoniae, and increases in AmpC $\beta$-lactamase-hyperproducing strains of Enterobacter, Citrobacter, and Serratia spp. In a Korean survey in 1998, which involved 25 hospitals, resistance rates of $E$. coli were $78 \%$ to ampicillin and $44 \%$ to cephalothin (Table 3 ). Cefotaxime resistance rates, determined by regular NCCLS breakpoint, were $8 \%$ for E. coli and $22 \%$ for K. pneumoniae. Therefore, ESBLproducing strains must be more prevalent than what is shown by the above rates. In a hospital, the rates of cefotaxime-susceptible $E$. coli and $K$. pneumoniae decreased from $99 \%$ to $89 \%$ and from $94 \%$ to $70 \%$, respectively, during the period 1986 to $1993 .^{26}$ Among the non-susceptible isolates, $69 \%$ of $E$. coli and $76 \%$ of $K$. pneumoniae were double-disk synergy-positive, indicating that they were ESBL producers. It was reported that ESBL- producing E. coli and K. pneumoniae were isolated only from nosocomially infected patients, which indicated that they were nosocomial pathogens. ${ }^{27}$ In a tertiary care hospital in 1999, resistance rates of isolates from inpatients and outpatients were: $E$. coli for cefotaxime, $23.6 \%$ vs $2.5 \%$; and ceftazidime, $7.9 \%$ vs $1.7 \%$; K. pneumoniae for cefotaxime, $17.5 \%$ vs $2.3 \%$; and ceftazidime, $31.7 \%$ vs $3.6 \%$. These findings suggest that the nosocomial spread of ESBLproducing strains is a significant cause of their high prevalence.

It was reported that, of five ESBL-producing isolates, four produced TEM-type and 1 produced SHV-type enzymes. ${ }^{28}$ Pai et al. $^{29}$ reported that the most prevalent ESBL types of $K$. pneumoniae, isolated at three university hospitals, were SHV-2, and SHV-12a, and TEM-52 types were reported later. It is interesting that Toho- and Kitasato-type ESBLs, found in Japan, have not yet been detected in Korea.

Since 1989, over 15 plasmid-encoded AmpC $\beta$ lactamases have been reported worldwide. ${ }^{30} \mathrm{~A}$ class $\mathrm{C} \beta$ lactamase, CMY-1, was first identified from K. pneumoniae in Korea in 1988. Bauernfeind et al ${ }^{31}$ reported that CMY$1 \mathrm{~b}$ had an amino acid change from Asn at position 346 of CMY-1a to Ile, resulting in increased resistance to ceftazidime. A survey in 1998 showed that $12 \%$ of $E$. coli and $14 \%$ of $\mathrm{K}$. pneumoniae were resistant to cefoxitin. ${ }^{5} \mathrm{Pai}$ et al. ${ }^{29}$ reported that AmpC $\beta$-lactamase was detected in 8 of $57 \mathrm{~K}$. pneumoniae isolates. Among the cefoxitin-resistant $E$. coli and K. pneumoniae, 6 of 19 and 3 of 7, respectively, had the conjugatively transferable CMY-1 $\beta$-lactamase gene. ${ }^{32}$

Enterobacter, Citrobacter, and Serratia spp. frequently cause nosocomial infections and are often resistant to third generation cephalosporins. In the 1998 survey, in Korea $47 \%$ and $51 \%$ of E. cloacae and S. marcescens, respectively, were resistant to cefotaxime (Table 3 ), ${ }^{5}$ resistance rates to ceftazidime were $48 \%$ and $25 \%$, respectively. Cefepime is

Table 3. Antimicrobial resistance rates of Enterobacteriaceae and glucose-nonfermenting gram-negative bacilli isolated in 1998 in Korea

\begin{tabular}{|c|c|c|c|c|c|c|}
\hline \multirow[t]{2}{*}{ Antimicrobial agents } & \multicolumn{6}{|c|}{ Percentage of isolates resistant (no. tested) ${ }^{\mathrm{a}}$} \\
\hline & $\begin{array}{l}\text { E. coli } \\
(20,604)\end{array}$ & $\begin{array}{l}\text { K. pneumoniae } \\
(9079)\end{array}$ & $\begin{array}{l}\text { E. cloacae } \\
(5781)\end{array}$ & $\begin{array}{l}\text { S. marcescens } \\
(3324)\end{array}$ & $\begin{array}{l}\text { A. baumannii } \\
(11866)\end{array}$ & $\begin{array}{l}\text { P. aeruginosa } \\
(20370)\end{array}$ \\
\hline Ampicillin & 78 & NT & NT & NT & NT & NT \\
\hline $\begin{array}{l}\text { Aminopenicillin/sulbactam } \\
\text { or clavulanic acid }\end{array}$ & 29 & 25 & 67 & 93 & 14 & NT \\
\hline Piperacillin & 61 & 35 & 54 & 38 & 75 & 43 \\
\hline Piperacillin/tazobactam & 5 & 10 & 35 & 37 & 43 & 35 \\
\hline Cephalothin & 44 & 38 & NT & NT & NT & NT \\
\hline Cefotaxime & 8 & 22 & 47 & 51 & 83 & NT \\
\hline Ceftazidime & 7 & 25 & 48 & 25 & 64 & 17 \\
\hline Cefoxitin & 12 & 14 & 97 & 74 & 98 & NT \\
\hline Cefotetan & 5 & 4 & 57 & 9 & 82 & NT \\
\hline Imipenem & 0 & 0 & 0.8 & 1 & 5 & 17 \\
\hline Amikacin & 5 & 9 & 16 & 20 & 60 & 30 \\
\hline Gentamicin & 32 & 28 & 47 & 48 & 79 & 50 \\
\hline Tobramycin & 25 & 32 & 51 & 62 & 78 & 48 \\
\hline Fluoroquinolone $^{\mathrm{a}}$ & 25 & 8 & 16 & 22 & 71 & 42 \\
\hline
\end{tabular}

NT, Not tested

${ }^{a}$ Tested either by ciprofloxacin, levofloxacin, or ofloxacin 
active against class $\mathrm{C} \beta$-lactamase-producing isolates. In 1991, it was reported that all isolates of Enterobacteriaceae tested were susceptible to cefepime ${ }^{33}$ and in 2000 in a tertiary care hospital, cefepime-resistant isolates remained rare, i.e., $1 \%$ each for E. coli and E. cloacae and $2 \%$ for $K$. pneumoniae and $4 \%$ for $S$. marcescens; none of the other species of Enterobacteriaceae were resistant.

Imipenem is the most active $\beta$-lactam drug against gram-negative bacilli, including $P$. aeruginosa and Acinetobacter baumannii. Imipenem-resistant $P$. aeruginosa and other gram-negative bacilli with IMP-1 $\beta$-lactamaseproduction were reported in Japan. ${ }^{34}$ This report was followed by others of carbapenemase-producing $P$. aeruginosa and $A$. baumannii in Italy, Greece, and France. ${ }^{35}$ In a Korean hospital, the imipenem-resistance rates of $P$. aeruginosa were $3 \%$ to $6 \%$ during the period 1994 1996 , but in the period $1997-1999$, the rates rose to $14 \%-$ $15 \%$. This tendency was also observed in other Korean hospitals. Some of this resistance was due to a decrease of outer membrane protein, but $8.7 \%$ of the imipenemresistant isolates produced carbapenemase. ${ }^{36}$ Interestingly, the polymerase chain reaction showed that the carbapenemase was not an IMP-type, but a VIM-type $\beta$ lactamase. It is of great concern that carbapenem resistance in Korea is increasing and that it is transferable by conjugation.

\section{Resistance of gram-negative bacilli to fluoroquinolones and aminoglycosides}

Ciprofloxacin, ofloxacin, and levofloxacin are fluoroquinolones that are very active against gram-negative bacilli, including enteric pathogens; however, increasing resistance is a worldwide concern. ${ }^{37}$ In a Korean hospital, only $5 \%$ of $E$. coli and $1 \%$ of $K$. pneumoniae were resistant to ofloxacin in 1991, but these rates rose rapidly, to $26 \%$ and $19 \%$, respectively, in 1994 . The resistance rate of $E$. coli to the fluoroquinolone was 33\% in 1999 (Fig. 2). The 1998 survey showed that resistance to the agent was more prevalent in large hospitals (defined as those with 1000 or more beds) compared with other hospital size categories), particularly in E. coli (Table 4). ${ }^{5}$ In a tertiary care hospital in 1999, the fluoroquinolone resistance rates of isolates from inpatients and outpatients were: E. coli, $39.8 \%$ vs $19.4 \% ; K$. pneumoniae, $13.8 \%$ vs $1.6 \%$. These results suggest the presence of nosocomial infections with fluoroquinolone-resistant $E$. coli and $K$. pneumoniae, together with existence of fluoroquinolone-resistant $E$. coli in the community. The fluoroquinolone resistance rates of $P$. aeruginosa and A. baumannii in the 1998 survey were $24 \%$ to $39 \%$ and $23 \%$ to $58 \%$, respectively, depending on the hospital size category.

The aminoglycoside-resistance pattern in the Far East was reported to differ from that in the United States. ${ }^{38}$ In 1998, the amikacin resistance rates of $E$. coli and $K$. pneumoniae in Korea were relatively low, at $5 \%$ and $9 \%$, respectively (Table 3), but the rates for E. cloacae and $S$. marcescens were $16 \%$ and $20 \%$, respectively, and rates were much higher for $A$. baumannii and $P$. aeruginosa, at $60 \%$ and $30 \%$, respectively. Gentamicin resistance rates were $32 \%$ for E. coli, $28 \%$ for K. pneumoniae, $48 \%$ for $S$. marcescens, $79 \%$ for $A$. baumannii, and $50 \%$ for P. aeruginosa. Tobramycin resistance rates were similar to those for gentamicin.

\section{Resistance of Salmonella and Shigella}

Ampicillin-resistant non-typhoidal Salmonella was rare in Korea until the early 1980s, i.e., only 4 of 211 isolates were resistant during the period $1979-1983$. In $1986,65 \%$ or more of Salmonella serovar Typhimurium were resistant to ampicillin, chloramphenicol, or tetracycline. ${ }^{39}$ In another study, $36 \%$ of Salmonella Typhimurium were resistant to ampicillin ${ }^{40}$ and this rate was similar to that in Japan. ${ }^{41}$ During the period 1995-1997, 5 strains of nontyphoidal Salmonella were isolated which produced TEM-52 ESBL.

Typhoid fever was prevalent until the early 1980s in Korea, but, since the early 1980s, the isolation of Salmonella Typhi has rapidly decreased and non-typhoidal Salmonella has increased. Antibiotic-resistant Salmonella Typhi was not known to exist until an isolate was detected in 1992 from a patient who had traveled to South East Asian countries. Two resistant isolates were again isolated at the same hospital in 1995 . In $1997,11 \%$ and $15 \%$ of the isolates collected by the National Institute of Health Korea were resistant to ampicillin and chloramphenicol, respectively. ${ }^{40}$

Bacillary dysentery was very prevalent in the past, but now it occurs much less frequently. It was reported that, among the Shigella strains isolated in $1998,8 \%$ were $S$. flexneri and $92 \%$ were $S$. sonnei. ${ }^{42}$ S. flexneri is well known for its antimicrobial resistance. Among the $S$. sonnei isolates in $1998,99 \%$ were resistant to cotrimoxazole and $71 \%$ were resistant to ampicillin.

\section{Resistance of Haemophilus influenzae to $\beta$-lactams}

H. influenzae is a frequent causative agent of bacterial meningitis. In the early 1980 s, $9 \%$ of $H$. influenzae isolates in Korea were resistant to ampicillin, by $\beta$-lactamase production. ${ }^{43}$ The rate gradually rose, and in the 1998 survey, it was $56 \%,{ }^{5}$ a rate that was much higher than that reported in the United States $\left(34 \%\right.$ in $\left.1993-1997^{44}\right)$ and that reported in Japan $\left(13.3 \%\right.$ in $\left.1996-1998^{45}\right)$. In the 1998 survey, $\beta$ lactamase-positivity rates of $H$. influenzae were similar in large and medium (less than 1000 beds) hospitals (Table 4). $\quad \beta$-Lactamase-negative ampicillin-resistant (BLNAR) strains and $\beta$-lactamase-positive aminopenicillin/ clavulanate-resistant (BLPACR) strains exist in Japan, but such strains have not been studied in Korea, as yet. 
Table 4. Comparison of resistance rates of some bacterial species, by hospital categories, in 1998 in Korea

\begin{tabular}{|c|c|c|c|c|c|}
\hline \multirow[t]{2}{*}{ Species } & \multirow[t]{2}{*}{$\begin{array}{l}\text { Antimicrobial } \\
\text { agents }\end{array}$} & \multicolumn{4}{|c|}{$\begin{array}{l}\text { Percentage of resistant isolates in hospital } \\
\text { categories }^{\mathrm{a}}\end{array}$} \\
\hline & & Large & $\begin{array}{l}\text { Seoul - } \\
\text { medium }\end{array}$ & $\begin{array}{l}\text { Non-Seoul - } \\
\text { Medium }\end{array}$ & Mean \\
\hline S. aureus & Oxacillin & 73 & 73 & 70 & 72 \\
\hline S. pneumoniae & Penicillin & 84 & 81 & 71 & 78 \\
\hline \multirow[t]{2}{*}{ E. faecium } & Ampicillin & 82 & 80 & 77 & 80 \\
\hline & Vancomycin & 8 & 14 & 10 & 11 \\
\hline E. faecalis & Vancomycin & 0.8 & 0.5 & 0.6 & 0.6 \\
\hline \multirow[t]{3}{*}{ E. coli } & Cefotaxime & 10 & 6 & 8 & 8 \\
\hline & Cefoxitin & 14 & 13 & 9 & 12 \\
\hline & Fluoroquinolones & 29 & 21 & 25 & 25 \\
\hline K. pneumoniae & Cefotaxime & 27 & 28 & 11 & 22 \\
\hline E. cloacae & Cefotaxime & 55 & 36 & 44 & 47 \\
\hline \multirow[t]{2}{*}{ P. aeruginosa } & Ceftazidime & 18 & 17 & 16 & 17 \\
\hline & Imipenem & 15 & 21 & 14 & 17 \\
\hline H. influenzae & Ampicillin & 60 & 58 & 56 & 58 \\
\hline
\end{tabular}

${ }^{\mathrm{a}}$ Hospital categories: large, $\geq 1000$ beds; medium, $<1000$ beds

\section{Resistance of Bacteroides fragilis}

Among the anaerobic bacteria, the Bacteroides species often cause infections. Only $1 \%$ of $B$. fragilis isolated in Korea in the period 1995-1996 showed resistance to cefoxitin, but the clindamycin resistance rate was $43 \%$, a rate much higher than that in other countries. ${ }^{46}$ During the period 1989-1996, none of the isolates of B. fragilis were resistant to imipenem, metronidazole, or chloramphenicol, but the resistance rates to piperacillin and cefotaxime during this period rose markedly, from $12 \%$ to $25 \%$, and from $17 \%$ to $33 \%$, respectively. In general, the resistance rates of the non-fragilis $B$. fragilis group species were higher than those of $B$. fragilis.

In summary, methicillin-resistant $S$. aureus, erythromycin-resistant $S$. pyogenes, penicillin nonsusceptible pneumococci, $\beta$-lactamase-producing gonococci, ESBL-producing E. coli and K. pneumoniae, class C $\beta$-lactamase-producing $E$. coli, fluoroquinolone-resistant E. coli, and aminoglycoside-resistant $A$. baumannii and $P$. aeruginosa are more prevalent in Korea, at higher rates than in other countries; this suggests the presence of high levels of antimicrobial selective pressure and the nosocomial spread of resistant bacteria. The recently observed rapid increases in vancomycin-resistant $E$. faecium and carbapenem-resistant $P$. aeruginosa present new threats in Korea.

\section{References}

1. Neu HC. The crisis in antibiotic resistance. Science 1992;257:106473.

2. Kunin CM. Resistance to antimicrobial drugs - a worldwide calamity. Ann Intern Med 1993;118:557-61.

3. National Committee for Clinical Laboratory Standards. Performance standards for antimicrobial susceptibility testing: eighth informational supplement. Wayne, PA: National Committee for Clinical Laboratory Standards; 1998.

4. Kim HO, Kang CK, Chong Y, Lee SY. Organisms isolated from blood at the Yonsei Medical Center, 1974-1983. Kor J Infect Dis 1985;17:15-31.

5. Lee K, Chang CH, Lee NY, Kim HS, Hong KS, Cho HC. Korean nationwide surveillance of antimicrobial resistance in 1998. Yonsei Med J 2000;41:497-506.

6. Lee MS, Chong Y. Characteristics of methicillin-resistant Staphylococcus aureus isolated from wounds in Korean patients. J Infect Chemother 1996;2:130-5.

7. Chong Y, Lee K, Shin JW, Shin HB, Lim JB. Activities of arbekacin against methicillin-resistant Staphylococcus aureus and Pseudomonas aeruginosa. J Kor Soc Chemother 1997;15:319-27.

8. Kim M-N, Pai CH, Woo JH, Ryu JS, Hiramatsu K. Vancomycinintermediate Staphylococcus aureus in Korea. J Clin Microbiol 2000;38:3879-81.

9. Lee K, Shin JW, Chong Y, Mikamo H. Trends in serotypes and antimicrobial susceptibility of group B streptococci isolated in Korea. J Infect Chemother 2000;6:93-7.

10. Okuyama M, Sagayama Y, Nakajima K. An epidemiological study of group A, B, C and $\mathrm{G}$ hemolytic streptococci isolated from elementary school children in the recent 12 years. Part II. Susceptibility to antibiotics. J Jpn Assoc Infect Dis 1994;68:665-79.

11. Uh Y, Jang IH, Yoon KJ, Lee $\mathrm{CH}$, Kwon JY, Kim MC. Colonization rates and serotypes of group B streptococci isolated from pregnant women in a Korean tertiary hospital. Eur J Clin Microbiol Infect Dis 1997;16:753-6.

12. Baquero F. Pneumococcal resistance to $\beta$-lactam antibiotics: a global geographic overview. Microb Drug Resist 1995;1:115-20.

13. Chong Y, Lee K, Kwon OH, Henrichsen J. Capsular types and antimicrobial resistance of Streptococcus pneumoniae isolated in Korea. Eur J Clin Microbiol Infect Dis 1995;14:528-31.

14. Lee H-J, Park J-Y, Jang S-H, Kim J-H, Kim E-C, Choi K-W. High incidence of resistance to multiple antimicrobials in clinical isolates of Streptococcus pneumoniae from a university hospital in Korea. Clin Infect Dis 1995;20:826-35.

15. Song JH, Yang JW, Peck KR, Kim S, Lee NY, Jacobs MR, et al. Spread of multi-resistant Streptococcus pneumoniae in South Korea. Clin Infect Dis 1997:25:747-9.

16. Ishida M, Watanabe H, Nagata M, Fukui Y, Ueda M, Furugo I, et al. Microbiological and clinical studies with Streptococcus pneumoniae isolated in five Kitakyushu municipal hospitals. J Jpn Assoc Infect Dis 1999;73:1116-22.

17. Lee HJ, Chong Y, Kwon OH. Species and antimicrobial susceptibility of Enterococcus isolated from clinical materials. Kor J Infect Dis 1992;24:115-20. 
18. Park SW, Lee SH, Choi HJ, Kim US, Kim NJ, Kim T, et al. Antibiotic susceptibility of enterococcal isolates causing bacteremia. Kor J Infect Dis 2000;32:227-32.

19. Park JW, Kim YR, Shin WS, Kang MW, Han KJ, Shim SI. Susceptibility tests of vancomycin-resistant enterococci. Kor J Infect Dis 1992;24:133-7.

20. Donskey CJ, Schreiber JR, Jacobs MR, Shekar R, Salata RA, Gordon S, et al. A polyclonal outbreak of predominantly VanB vancomycin-resistant enterococci in Northeast Ohio. Clin Infect Dis 1999;29:573-9.

21. Kim J-H, Ahn J-G, Jeong S-J, Kim Y-T, Kim J-W, Kim S-Y, et al. Prevalence of penicillinase-producing Neisseria gonorrhoeae in Seoul (1994). J Kor Soc Chemother 1996;14:107-11.

22. Lee K, Shin JW, Lim JB, Kim YA, Yong D, Oh H-B, et al. Emerging antimicrobial resistance, plasmid profile and pulsedfield gel electrophoresis pattern of the endonuclase-digested genomic DNA of Neisseria gonorrhoeae. Yonsei Med J 2000;41:381-6.

23. Kam KM, Lo KK, Ho NKY, Cheung MM. Rapid decline in penicillinase-producing Neisseria gonorrhoeae in Hong Kong associated with emerging 4-fluoroquinolone resistance. Genitourin Med 1995;71:141-4.

24. Tanaka M, Naito S, Nakayama H, Kobayashi I. Antimicrobial susceptibility of Neisseria gonorrhoeae in Fukuoka City, Japan in the early 1980s and 1997-1998: emergence of high-level fluoroquinolone resistance. Antimicrob Agents Chemother 1999;43:7223 .

25. WHO Western Pacific Region Gonococcal Antimicrobial Surveillance Programme. Surveillance of antibiotic susceptibility of Neisseria gonorrhoeae in the WHO Western Pacific Region 1992-1994. Genitourin Med 1997;73:355-61.

26. Lee K, Cho SR, Lee CS, Chong Y, Kwon OH. Prevalence of extended broad-spectrum $\beta$-lactamase in Escherichia coli and Klebsiella pneumoniae. Kor J Infect Dis 1994;26:341-8.

27. Choi YH, Lee SM, Park KJ, Hwang SC, Lee YH, Hahn MS. A comparative study of community-acquired Klebsiella pneumoniae bacteremia and Escherichia coli bacteremia. Kor J Infect Dis 2000;32:197-202.

28. Chong Y, Lee K, Okamoto R, Inoue M. Characteristics of extended-spectrum $\beta$-lactam hydrolyzing activity of Klebsiella pneumoniae and Escherichia coli strains isolated from clinical specimens. Kor J Infect Dis 1997;29:477-85.

29. Pai H, Kim JM, Kwon YM, Lee K, Chong Y, Kim EC, et al Characterization of extended-spectrum $\beta$-lactamases in Klebsiella pneumoniae isolated in Korea. J Kor Soc Infect Dis 1977;29:93103.

30. Bauernfeind A, Chong Y, Schweighart S. Extended broad spectrum $\beta$-lactamase in Klebsiella pneumoniae including resistance to cephamycins. Infection 1989;17:316-21.

31. Bauernfeind A, Chong Y, Lee K. Plasmid-encoded AmpC $\beta$ lactamase: how far have we gone 10 years after the discovery? Yonsei Med J 1998;39:520-5.
32. Kim J, Kwon Y. AmpC-type $\beta$-lactamase in clinical isolates of cefoxitin-resistant Escherichia coli and Klebsiella pneumoniae. J Kor Soc Microbiol 1999;34:327-36.

33. Chong Y, Lee K, Kwon OH. In-vitro activities of cefepime against Enterobacter cloacae, Serratia marcescens, Pseudomonas aeruginosa and other aerobic gram-negative bacilli. J Antimicrob Chemother 1933; 32(Suppl B):21-9.

34. Watanabe M, Iyobe S, Inoue M, Mitsuhashi S. Transferable imipenem resistance in Pseudomonas aeruginosa. Antimicrob Agents Chemother 1991;35:147-51.

35. Poirel L, Naas T, Nicolas D, Collet L, Bellais S, Cavallo J-D, et al. Characterization of VIM-2, a carbapenem-hydrolyzing metallo- $\beta$ lactamase and its plasmid- and integron-borne gene from a Pseudomonas aeruginosa clinical isolate in France. Antimicrob Agents Chemother 2000;44:891-7.

36. Lee K, Chong Y, Shin HB, Yong D. Rapid increase of imipenemhydrolyzing Pseudomonas aeruginosa in a Korean hospital. 38th ICAAC, Abstract E-85 Washington, DC: American Society for Microbiology 1998.

37. Chong Y, Lee K. Review of quinolone resistance in human pathogens. WHO meeting on the use of quinolones in food animals and potential impact on human health. WHO, Geneva, 2-5 June 1998.

38. Shimizu K, Kumada T, Hsieh W-C, Chung H-Y, Chong Y, Hare $\mathrm{RS}$, et al. Comparison of aminoglycoside resistance patterns in Japan, Formosa, and Korea, Chile, and the United States. Antimicrob Agents Chemother 1985;28:282-8.

39. Chong Y, Han SS, Kwon OH, Lee SY, Jung TH. Increased isolation of ampicillin- and chloramphenicol-resistant Salmonella typhimurium. J Kor Soc Microbiol 1987;22:55-9.

40. Shin YH, Yoo JS, Kim KS, Chung DJ, Oh KS, Lee JK, et al. Invitro antimicrobial susceptibility of Salmonella typhi, Salmonella typhimurium and Salmonella enteritidis isolated in Korea, 1997. J Kor Soc Chemother 1998;16:205-14.

41. Matsushita S, Konishi N, Arimatsu M, Kai A, Yamada S, Morozumi S, et al. Drug resistance and definitive type 104 of Salmonella serovar Typhimurium isolated from sporadic cases in Tokyo, 1980-1998. J Jpn Assoc Infect Dis 1999;73:1087-94.

42. National Institute of Health Korea. Bacterial dysentery. Communicable Diseases Monthly Report 1999;10:49-55.

43. Chong Y, Lee SY. Ampicillin and cefaclor susceptibility of Haemophilus influenzae. J Kor Soc Chemother 1985;3:142-8.

44. Derecola A, Butler DL, Kaplan RL, Miller LA, Poupard JA. A 5year surveillance study of 44691 isolates of Haemophilus influenzae project Beta-Alert 1993-1997. Antimicrob Agents Chemother 1999;43:185-6.

45. Watanabe N, Niki W, Matsushima T, Watanabe N, Niki H, Matsushima T. Antimicrobial resistance in Haemophilus influenzae. J Jpn Assoc Infect Dis 1999;73:494.

46. Lee K, Shin HB, Chong Y. Antimicrobial resistance patterns of Bacteroides fragilis group organisms in Korea. Yonsei Med J 1998;39:578-86. 